\title{
Energy-Efficient Connected Coverage of Discrete Targets in Wireless Sensor Networks ${ }^{\star}$
}

\author{
Mingming $\mathrm{Lu}^{+}, \mathrm{Jie} \mathrm{Wu}^{+}$, Mihaela Cardei ${ }^{+}$, and Minglu $\mathrm{Li}^{*}$ \\ ${ }^{+}$Department of Computer Science and Engineering \\ Florida Atlantic University, USA \\ ${ }^{*}$ Department of Computer Science and Engineering \\ Shanghai Jiao Tong University, China
}

\begin{abstract}
A major concern in wireless sensor networks is to maximize network lifetime (in terms of rounds) while maintaining a high quality of services (QoS) at each round such as target coverage and network connectivity. Due to the power scarcity of sensors, a mechanism that can efficiently utilize energy has a great impact on extending network lifetime. Most existing works concentrate on scheduling sensors between sleep and active modes to maximize network lifetime while maintaining target/area coverage and network connectivity. This paper generalizes the sleep/active mode by adjusting sensing range to maximize total number of rounds and presents a distributed heuristic to address this problem.
\end{abstract}

\section{Introduction}

The paramount concern in wireless sensor networks (WSNs) is power scarcity, driven partially by battery size and weight limitations. Mechanisms that optimize sensor energy utilization have a great impact on extending network lifetime. Power saving techniques can generally be classified in two categories: scheduling sensors to alternate between active and sleep mode, or adjusting their sensing ranges. In this paper, we combine both methods by dynamic management of node duty cycles in a high target density environment. In this approach, any sensor schedules its sensing ranges from 0 to its maximum range, where range 0 corresponds to sleep mode.

Target coverage characterizes the monitoring quality of WSNs. The general requirement of target coverage is that each target should be covered by at least one sensor. The energy consumption of target coverage is the total energies consumed by all sensors. The problem of the single sensing range is that there exists a lot of targets covered by several active sensors together, which causes redundancy in energy consumption. Adjustable sensing ranges [16] allow sensors more choices to reduce their energy consumption, and thus prolong WSNs' lifetime.

However, target coverage is not the only responsibility of WSNs. To reduce network overhead and energy consumption, WSNs should also provide satisfactory network connectivity so that sensors can communicate for data gathering or data fusion.

\footnotetext{
* The work was supported in part by NSF grants ANI 0083836, CCR 0329741, CNS 0422762, CNS 0434533, EIA 0130806, NSFC (No. 60473092), and Program for New Century Excellent Talents in University (No. NCET-04-0392).Email: \{mlu2@, jie@cse., mihaela@cse.\}fau.edu, mlli@sjtu.edu.cn
} 
In this paper, we study the problem of maximizing network lifetime (in terms of rounds) in WSNs, where in each round, sensor-target coverage and sensor connectivity are maintained. Unlike the traditional approaches [12], [14] in area coverage where the connectivity is trivialized by assuming that the transmission range is at least twice of the sensing range, we focus on a more generic connectivity condition that can be used even when the transmission range is less than twice the sensing range.

Although maximizing the lifetime of WSNs by scheduling sensors' activity is not a new problem, none of existing algorithms deal with the case of scheduling sensors' activity by self-configuring sensing ranges, in the environment where both discrete target coverage and network connectivity are satisfied.

The main contributions of this paper are: 1) to introduce the adjustable sensing range connected sensor cover (ASR-CSC) problem, where target coverage and connectivity are maintained, 2) to present a generic connectivity condition, 3) to design efficient distributed heuristics to solve the ASR-CSC problem, 4) to demonstrate the performance of our approach through simulations.

The rest of the paper is organized as follows. In section 2 we present related works on coverage and connectivity problems. Section 3 formulates the ASR-CSC problem and section 4 presents our heuristic contributions. In section 5 we present the simulation results and section 6 concludes our paper.

\section{Related Work}

The general target coverage problem is introduced in [1], where the problem is modelled as finding maximal number of disjoint set covers, such that every cover completely monitors all targets. The general problem is NP-complete [1]. This problem is extended further in [2], where sensors are not restricted to participation in only disjoint sets, i.e. a sensor can be active in more than one set.

Authors in [15] study area coverage and connectivity in an unreliable wireless sensor grid network, and present a necessary and sufficient condition for coverage and connectivity. In [14], a sufficient condition, the transmission range being larger than twice the sensing range, under which coverage implies connectivity, is given. A similar sufficient condition is considered in [12] in the environment that requires target coverage and connectivity of active sensors in a large scale WSN. Although the connectivity can be relatively easy to specify in the environment with area coverage and uniform sensing range, such a condition will be hard to specify in the environment with with adjustable sensing range and discrete target coverage. In this paper, we present a generic way to address this problem.

The work most relevant to our approach is [3], which extends [2] with adjustable sensing range in point coverage (where target are discrete). Compared with [3], we are also concerned with maintaining network connectivity for the ASR-CSC problem. We analyze the impact of connectivity on energy efficient management sensors, present a generic connectivity condition, and design a distributed heuristic algorithm to maximize the lifetime of WSNs. 


\section{Problem Formulation}

We have two important assumptions in this paper: 1) all sensors in WSNs are connected; 2)any target should be located in the maximal sensing range of at least one sensor. In this paper, we compute the sensor-target coverage and sensor-sensor connection relationship based on Euclidean distance, i.e., a sensor covers a target with sensing range $r_{k}$ if the Euclidean distance between them is no greater than $r_{k}$, and sensor $i$ is connected to sensor $j$ if their Euclidean distance is no greater than transmission range $r_{c}$. In this paper, we adopt a fixed transmission range $r_{c}$ and adjustable sensing ranges $R=\left\{r_{0}, r_{1}, \cdots, r_{k}, \cdots, r_{P}\right\}$, in which $r_{k}$ is the $k$-th sensing range. In particular, $r_{0}=0$ is 0 -th sensing range, corresponding to sleep mode, $r_{1}$, the minimum sensing range in active mode, is the 1 -st sensing range, and $r_{P}$ the maximum sensing range, is the $P$-th sensing range. For convenience, we index sensor $i$ 's selected sensing range by $p(i)$, and $p(i)=k$ means sensor $i$ 's current sensing range is the $k$ th range $r_{k}$. For consistance, we use $R_{c}$ to denote the transmission range set, i.e., $R_{c}=\left\{r_{c}\right\}$. We denote $S, T$ to be the set of sensors and the set of targets respectively, in which $s_{i} \in S$ means sensor $i$, and $t_{j} \in T$ represents target $j$. Finally, we define $S(i)$ the sensors within $s_{i}$ 's transmission range.

Upon above notations, we model our problem on graph $G_{U} \cup G_{D}$, where $G_{U}=$ $\left(S, R_{c}, E_{S}\right)$ is the sensor communication graph, and $G_{D}=\left(S \cup T, R, E_{D}\right)$ is the sensor-target coverage graph. $G_{U}$ is undirected since sensors' communication ranges are the same, and $G_{D}$ is directed since different sensors can set different sensing ranges. $E_{S}=\left\{\left(s_{i}, s_{j}\right)|| s_{i} s_{j} \mid \leq r_{c}\right\}$ is a subset of $S \times S$, which characterizes the direct connection between any two sensors. $E_{D}=\left\{\left(s_{i}, r_{p(i)}, t_{j}\right)|| s_{i} t_{j} \mid \leq r_{p(i)}\right\}$ is a subset of $S \times R \times T$, which represents the sensor-target coverage relationship. Triple $\left(s_{i}, r_{p(i)}, t_{j}\right)$ means sensor $s_{i}$ with sensing range $r_{p(i)}$ covering target $t_{j}$. Let $S_{a}=$ $\left\{s_{i} \mid p(i)>0, \forall s_{i} \in S\right\}$ be the active sensors in each round. Target coverage can be defined: at any given time during the lifetime of WSNs, $\forall t_{j} \in T, \exists s_{i} \in S_{a}$ such that $\left(s_{i}, r_{p(i)}, t_{j}\right) \in E_{D}$. WSNs' connectivity depends on the connectivity of its communication graph $G_{U}$, thus we can adopt the following definition, network connectivity: $\forall s_{i}, s_{j} \in S_{a}, \exists s_{i_{1}}, s_{i_{2}}, \cdots, s_{i_{m}} \in S_{a}$, such that $\left(s_{i}, s_{i_{1}}\right),\left(s_{i_{1}}, s_{i_{2}}\right), \cdots,\left(s_{i_{m}}, s_{i_{j}}\right)$ $\in E_{S}$. Thus, our problem can be formally defined as follows:

Definition 1. (ASR-CSC Problem) Given a set of targets and a set of sensors with adjustable sensing ranges in a WSN, schedule sensors' sensing ranges, such that the WSN's lifetime is maximized, under the conditions that both target coverage and network connectivity are satisfied, and each sensor's energy consumption should be no more than initial energy $E$.

There are two energy models in this paper. The first model is linear model, in which energy consumption is a linear function of the sensing range. The second model is quadratic model, in which energy consumption is a quadratic function of the sensing range. We do not consider the energy consumption caused by transmission. We denote $e_{k}=f\left(r_{k}\right)$ the energy consumption under sensing range $r_{k}$, in which $f$ can be linear or quadratic. A comparison of these two models is illustrated in section 5 . 
Since AR-SC problem [3] is a special case of the ASR-CSC problem by assuming the communication graph $G_{U}$ to be a complete graph, according to restriction method [6], the ASR-CSC problem is NP-complete.

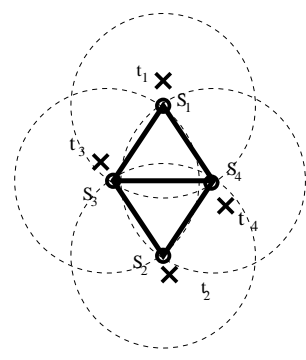

(a)

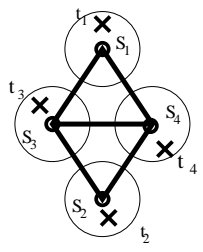

(c)

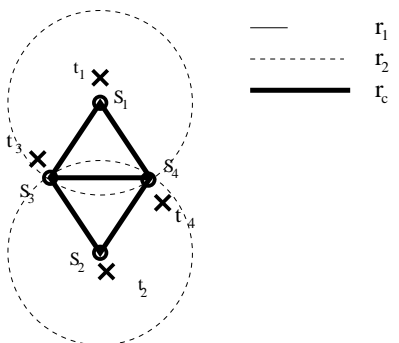

(b)

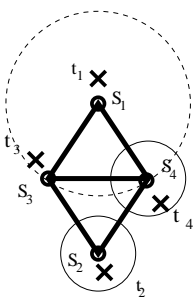

(e)

Fig. 1. Example of connected sensor covers

Figure 1 shows an example with four sensors $s_{1}, s_{2}, s_{3}, s_{4}$ and four targets $t_{1}, t_{2}, t_{3}$, $t_{4}$. In this example we assume a sensor's sensing area is a disk centered at the sensor, with a radius equal to the sensing range. Each sensor has two sensing ranges $r_{1}, r_{2}$ with $r_{1}<r_{2}$. We use circles with solid lines to denote sensing area with range $r_{1}$, circles with dotted lines for area with range $r_{2}$, and heavy solid lines for transmission range $r_{c}$. The sensor-target coverage relationships are illustrated in Figure 1 (a), (c). Figure 1 (c) shows the targets covered by each sensor with range $r_{1}:\left(s_{1}, r_{1}\right)=\left\{t_{1}\right\},\left(s_{2}, r_{1}\right)=$ $\left\{t_{2}\right\},\left(s_{3}, r_{1}\right)=\left\{t_{3}\right\}$, and $\left(s_{4}, r_{1}\right)=\left\{t_{4}\right\}$. Figure 1 (a) shows the targets covered by each sensor with range $r_{2}:\left(s_{1}, r_{2}\right)=\left\{t_{1}, t_{3}\right\},\left(s_{2}, r_{2}\right)=\left\{t_{2}, t_{4}\right\},\left(s_{3}, r_{2}\right)=\left\{t_{3}\right\}$, and $\left(s_{4}, r_{2}\right)=\left\{t_{4}\right\}$. The sensors' connection relationships are presented in solid lines: $S\left(s_{1}\right)=\left\{s_{3}, s_{4}\right\}, S\left(s_{2}\right)=\left\{s_{3}, s_{4}\right\}, S\left(s_{3}\right)=\left\{s_{1}, s_{2}, s_{4}\right\}, S\left(s_{4}\right)=\left\{s_{1}, s_{2}, s_{3}\right\}$.

All possible connected sensor covers $C_{1}, C_{2}, C_{3}$ are illustrated in Figure 1 (c), (d), and (e) respectively, where $C_{1}=\left\{\left(s_{1}, r_{1}\right),\left(s_{2}, r_{1}\right),\left(s_{3}, r_{1}\right),\left(s_{4}, r_{1}\right)\right\}, C_{2}=\left\{\left(s_{1}, r_{1}\right)\right.$, $\left.\left(s_{2}, r_{2}\right),\left(s_{3}, r_{1}\right)\right\}$, and $C_{3}=\left\{\left(s_{1}, r_{2}\right),\left(s_{2}, r_{1}\right),\left(s_{4}, r_{1}\right)\right\}$. Figure 1 (b) shows an sensor cover which doesn't meet the connectivity requirement.

In this example, we assume $E=2, e_{1}=0.5$, and $e_{2}=1$. Each set cover is active for a unit time of 1 . The optimal solution has the following sequence of sensor covers: $C_{1}, C_{1}, C_{1}, C_{1}$ with maximum lifetime 4 . After that, all sensors run out of energy.

If sensors do not have adjustable sensing ranges and the sensing range equal to $r_{2}$, then all sensors should be active. The reason is that $s_{1}$ and $s_{2}$ have to be active to cover 
$t_{1}$ and $t_{2}$, and one of $s_{3}$ and $s_{4}$ has to be active to maintain connectivity. Sensors can be organized in two distinct set covers, i.e., $C_{4}=\left\{s_{1}, s_{2}, s_{3}\right\}$ and $C_{5}=\left\{s_{1}, s_{2}, s_{4}\right\}$. But no matter how we schedule the set of sensors, the life time can be no more than 2. Therefore, this example shows a $100 \%$ lifetime increase when adopting adjustable

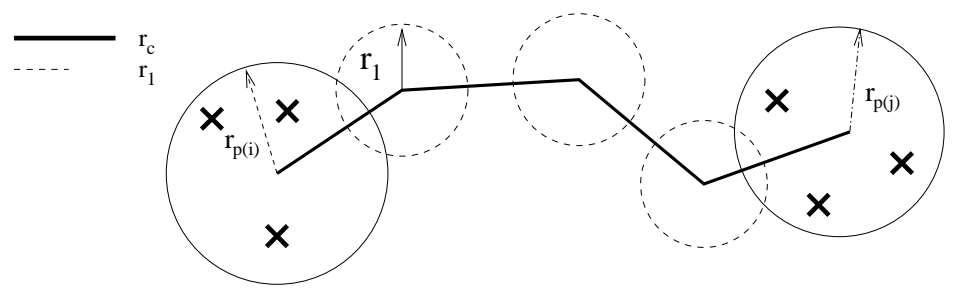

Fig. 2. Sensors contribute only for connectivity

sensing ranges.

\section{Solution for the ASR-CSC Problem}

In this section, a distributed and localized algorithm is given to solve the ASR-CSC problem. In the traditional area coverage, the connectivity is ensured if $r_{c} \geq 2 \cdot r_{k}$ for the case of uniform sensing range $r_{k}$. However, this result does not apply to point coverage even when $r_{k}=r_{P}$. A simple illustration is shown in Figure 2, where heavy solid lines represent transmission range $r_{c}$ and circles with with light dotted lines denote sensing area with the minimal sensing range $r_{1}$. Two sensors $i$ and $j$ with sensing ranges $r_{p(i)}$ and $r_{p(j)}$ respectively take the responsibility of covering discrete targets. However, $i$ and $j$ are so far apart that a range $r_{c}\left(\geq 2 \cdot r_{1}\right)$ cannot connect $i$ and $j$. Therefore, we have to select some sensors not for target coverage but for connecting $i$ and $j$. In this case, three other sensors have to be active just for connectivity. The sensing ranges of the three interconnected sensors are $r_{1}$ in order to save energy while maintaining connectivity. In fact, $r_{1}$ can be considered the minimal energy consumption of an active sensor.

Instead of narrowing our efforts on the relationship between target coverage and network connectivity, we focus on finding a generic way to satisfy both discrete target coverage and network connectivity. We build a virtual backbone first to satisfy network connectivity, and ensure coverage based on that backbone.

We first give a high level view of the whole algorithm. Our algorithm works in rounds, at the beginning of each round the following steps execute: 1) Construct a virtual backbone for the WSN; 2) For each sensor in the virtual backbone, set its sensing range to be the minimal range $r_{1}$;3) All remaining sensors with range $r_{0}$ (dominatees) together with sensors with range $r_{1}$ (dominators) iteratively adjust their sensing ranges based on contribution (the ratio of the number of covered targets to $e_{p(i)}$, corresponding to $\left.r_{p(i)}\right)$ until a full coverage is found; 4) Each active sensor $i$ reduces $e_{p(i)}$ from its residual energy. 
In providing such a virtual backbone in our algorithm, we first construct a connected dominating set and prune redundant sensors by applying Rule- $k$ in [13]. Since it is a distributed and localized method, to ensure network connectivity, we have to assume that the sensors in a given area are dense enough so that all sensors in that area are connected. However, target need not to be dense.

In this method, each sensor determines its status (active/sleep) by applying an eligibility rule. If it meets the rule's requirement, then it decides to sleep; otherwise, it chooses to work for the rest of the round. We formally define the rule : let $S_{h}(i)$ be the sensors in $S(i)$ (Note $S(i)$ is $i$ 's neighbor sensors) with higher priority than $i$ 's. $i$ is able to sleep if and only if the following conditions are satisfied: 1) Sensors in $S_{h}(i)$ are connected. 2) Sensor $i$ 's low priority neighbor $S(i)-S_{h}(i)$ are covered by sensors in $S_{h}(i)$.

The result of this connectivity initialization phase is the set of connected active sensors (dominators). The sensing range of those sensors will be set to $r_{1}$ in order to save energy. Since dominators alone cannot guarantee target coverage, all dominators together with all still inactive sensors (dominatees) will participate in a coverage initialization phase to ensure target coverage. The reason for active sensors participating in the coverage initialization phase is that dominators can contribute more than dominatees. Since some targets can be located in a distant location such that no dominators can cover those targets, so dominatees should participate the coverage initialization phase.

We present the connectivity initialization phase. This phase is run by each individual sensor before the coverage initialization phase.

\section{Connectivity Initialization}

1: start a timer $t_{i} \leftarrow \frac{W}{b(i)}$

2: if receiving message from $s_{j}$ before $t_{i}$ expires then

3: $\quad S_{h}(i) \leftarrow S_{h}(i) \cup j$;

4: $\quad$ Construct subgraph $\left(S(i), E_{S(i)}\right)$;

5: $\quad$ if $S_{h}(i)$ is connected and covers $S(i)-S_{h}(i)$ then

6: $\quad p(i) \leftarrow 0$;

7: $\quad$ end if

8: end if

9: $p(i) \leftarrow 1$

In the above algorithm, $b(i)$ denotes the residual energy of sensor $i, S_{h}(i)$ represents sensor $i$ 's neighbor sensors, which have higher residual energy than that of $i$ or have higher ID when residual energies are equal, and $W$ is the longest back-off time. Assigning higher priority to higher residual energy sensors is to balance energy consumption among sensors in the virtual backbone.

In forming the virtual backbone, each sensor $i$ self determines its responsibility by testing Rule- $k$. If it is satisfied, $i$ decides to sleep; otherwise, it chooses to work. After the connectivity initialization phase, all dominators will be active for the rest of the round. But $r_{1}$ is not the final sensing ranges for dominators. The dominators can adjust their sensing range if more contributions can be obtained than other sensors'. After the connectivity initialization phase, a second phase is issued to guarantee target coverage. In the second phase, dominatees combined with dominators will jointly take the responsibility to ensure target coverage, and a sensor's sensing range is increased 
based on its contribution to target coverage. Once the second phase is done, the sensors whose sensing range greater than $r_{0}$ will form the connected sensor cover, while all other sensors will be off-duty in the current round.

To complete our algorithm, we informally describe the coverage initialization phase. For the coverage initialization phase, We use a distributed algorithm similar to the one in [4] to handle target coverage. For brevity, we just describe the main idea of the target coverage algorithm. In each round, each sensor $i$ backs off a time in reverse propor-

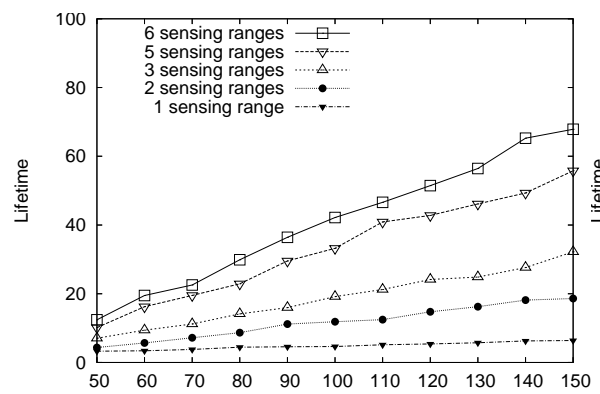

(a)

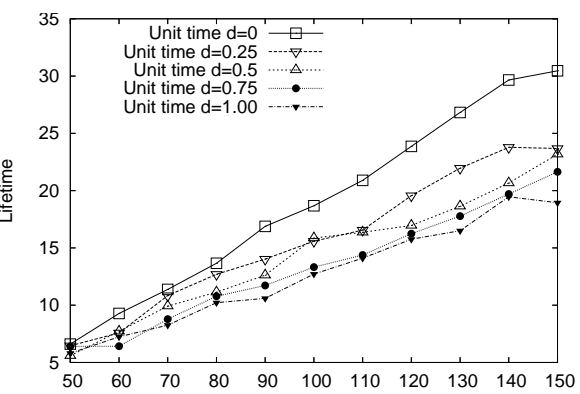

(b)

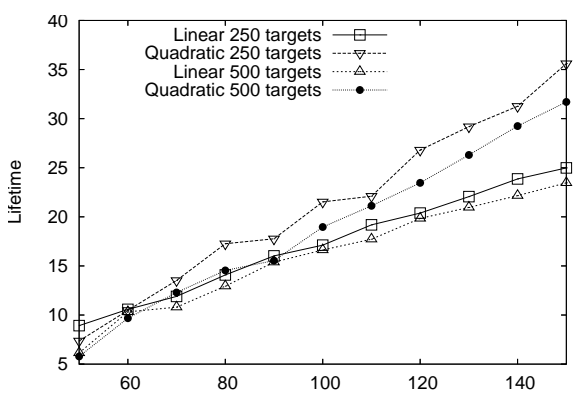

(c)

Fig. 3. Simulation results

tion to its maximal contribution. If before the back-off time is up, it receives messages from its neighbors, it reduces its uncovered target set, recalculates its contribution, and adjusts its back-off time. When the back-off time is up, it broadcasts $p(i)$ (that corresponds to the maximal contribution) and covered target set to its neighbors. At the end of this stage, all the targets will be covered.

\section{Simulation Results}

In this section, we give an evaluation of our distributed algorithm. Our simulations are based on a stationary network with sensor nodes and targets randomly located in a $100 m \times 100 m$ area. We assume sensors are homogeneous and initially have the same 
energy. In the simulation, we consider the following tunable parameters: 1) the number of sensor nodes $N$. In our experiments we vary it between 50 and 150;2) the number of targets to be covered $M$. It varies it between 250 to $500 ; 3$ ) the number of positive sensing ranges $P$. We vary it between 1 and 6 , and the sensing range values between $10 \mathrm{~m}$ and $60 \mathrm{~m} ; 4$ ) Time slot $d$, which shows the impact of the transfer delay on the performance of the distributed greedy heuristic. We vary $d$ between 0 and 1 with increase 0.25 .

In the first experiment in Figure 3(a), we study the impact of the number of adjustable sensing ranges on network lifetime. We consider 500 targets randomly distributed and we vary the number of sensors between 50 and 150 with an increment of 10 . We let the largest sensing range be $30 \mathrm{~m}$ for all cases. We observe the network lifetime when sensors support up to 6 sensing range adjustments: $r_{1}=5 \mathrm{~m}, r_{2}=10 \mathrm{~m}$, $r_{3}=15 \mathrm{~m}, r_{4}=20 \mathrm{~m}, r_{5}=25 \mathrm{~m}$, and $r_{6}=30 \mathrm{~m}$. A case with $P$ positive sensing ranges, where $P=1 . .6$, allows each sensor node to adjust $P+1$ sensing ranges $r_{0}$, $r_{1}, r_{2}, \cdots, r_{P}$. Note that $P=1$ is the case when all sensor nodes have a fixed sensing range with value $20 \mathrm{~m}$. The other environment parameters include initial energy 20 . Simulation results indicate that adjustable sensing ranges have great impact on network lifetime.

In Figure 3(b) we observe the network lifetime under different unit time assumptions. We measure the network lifetime when the number of sensors varies between 50 and 150 with an increment of 10 and the number of targets is 500. Each sensor has 3 sensing ranges with values $10 \mathrm{~m}, 20 \mathrm{~m}$, and $30 \mathrm{~m}$. The energy consumption model is quadratic. We change the length of the unit time $d$ in the distributed greedy algorithm to $d=0, d=0.25,0.5,0.75$ and 1 . Network lifetime produced by the algorithm with lower unit time is longer than those with higher unit time. This happens because, in the distributed heuristic, breaking a tie is at the expense of back-off time, and there is also no guarantee of avoid conflict. A conflict occurs the time between any two sensors' broadcast is less than $d$. Then, there might be sensors that work instead of going to the sleep state, even if the targets within their sensing ranges are already covered. As illustrated in Figure 3(b), the transfer delay also affects the network lifetime. The longer the transfer delay, the shorter the lifetime.

In Figure 3(c) we study the impact of two energy models on the network lifetime computed by the distributed greedy heuristic when we vary the number of sensors between 50 and 150, and the number of targets is 250 or 500 . Each sensor has $P=3$ sensing ranges with values $10 \mathrm{~m}, 20 \mathrm{~m}$, and $30 \mathrm{~m}$. The two energy models are the linear model $e_{p}=c_{1} * r_{p}$, and quadratic model $e_{p}=c_{2} * r_{p}^{2}$. In this experiment we defined constants $c_{1}=E / 2\left(\sum_{r=1}^{P} r_{p}\right)$ and $c_{2}=E / 2\left(\sum_{r=1}^{P} r_{p}^{2}\right)$, where $E=20$ is the sensor starting energy. For both energy models, the simulation results are consistent and indicate that network lifetime increases with the number of sensors and decreases as more targets have to be monitored.

In Figure 4, we give an example of active sensor set in a round. We assume a $100 \mathrm{~m} \times$ $100 \mathrm{~m}$ area, with 40 sensors and 25 targets. We use a linear energy model. The first graph represents the sensors' and targets' random deployment. The transmission range $r_{c}$ is $25 \mathrm{~m}$. If the distance between any two sensor nodes is no more than $r_{c}$, we connect these two sensors by a undirected link. Thus a connected graph is constructed, as shown in 


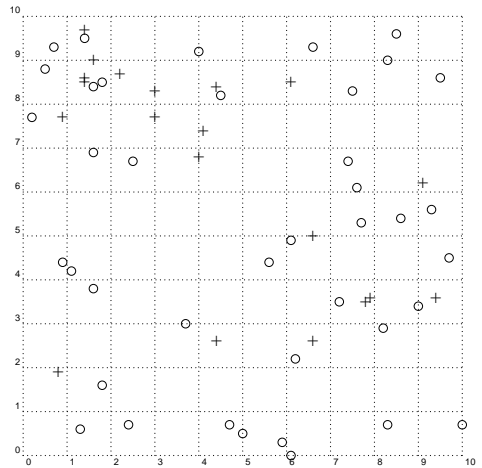

(a)

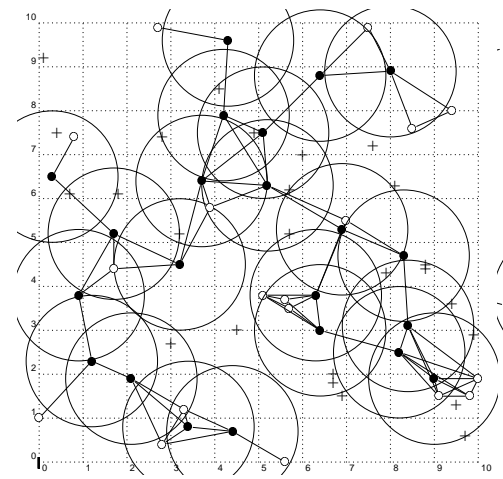

(c)

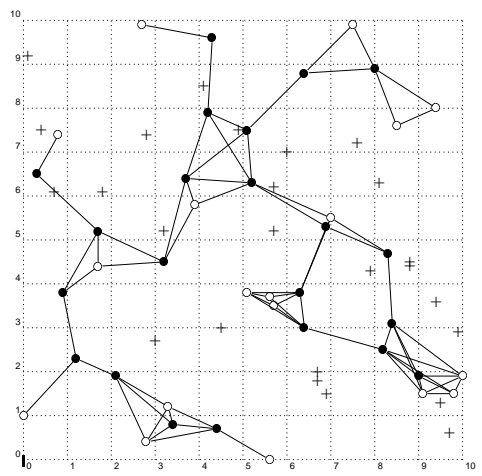

(b)

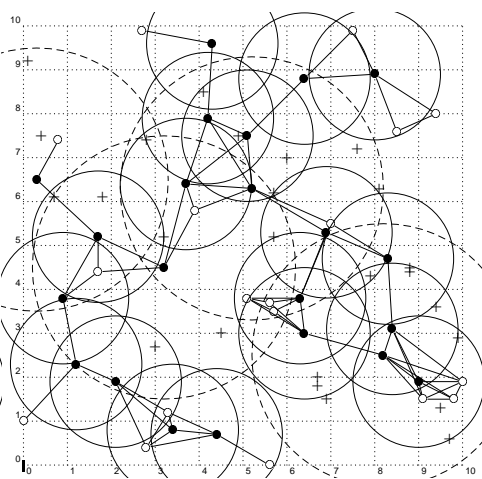

(d)

Fig. 4. Set covers example, where " $\circ^{\prime \prime}$ are sensors and $"{ }^{\prime \prime}$ are targets. (a) Sensors and targets deployment. (b) Connected dominating set (black nodes) selected by Connectivity Initialization. (c) Partial coverage when all sensors in the connected dominating set work in sensing range $r_{1}$. (d) Full coverage.

4 (b). Notice that the active sensors are blackened. Each sensor has $P=3$ sensing ranges with values $15 \mathrm{~m}, 30 \mathrm{~m}$, and $45 \mathrm{~m}$. We use solid lines to represent $r_{1}=15 \mathrm{~m}$, dashed lines for $r_{2}=30 \mathrm{~m}$, and dotted lines for $r_{3}=45 \mathrm{~m}$. Figure 4 (c) show a partial coverage when connected dominating sensors, which are selected in the connectivity initialization phase, keep sensing range $r_{1}$. Figure 4(d) shows the schedule satisfying both connectivity and coverage. Note the line type indicates the sensing range value.

\section{Conclusions}

In this paper, we study the problem to maximize WSN's lifetime (in terms of rounds) while maintaining both discrete target coverage and network connectivity. This not only provides satisfied quality of service in WSNs, but also presents more options and chal- 
lenges to design an energy efficient sensor scheduling. We study the relationship between network connectivity and target coverage and introduce a generic condition to guarantee network connectivity. We design a round-based distributed algorithm to coordinately determine sensors' sensing range based on different relations between transmission range and maximal sensing range.

In the future, we will study the impact of the degree of coverage on network lifetime and its relationship with network connectivity. We will also take into account the communication cost and its impact on network lifetime.

\section{References}

1. M. Cardei, D.-Z. Du, Improving Wireless Sensor Network Lifetime through Power Aware Organization, ACM Wireless Networks, Vol 11, No 3, pg. 333-340, May 2005.

2. M. Cardei, M. Thai, Y. Li, and W. Wu, Energy-Efficient Target Coverage in Wireless Sensor Networks, IEEE INFOCOM 2005, Mar. 2005.

3. M. Cardei, J. Wu, M. Lu, and M. Pervaiz, Maximum Network Lifetime in Wireless Sensor Networks with Adjustable Sensing Ranges, IEEE WiMob2005, Aug. 2005.

4. M. Cardei, J. Wu, Energy-Efficient Coverage Problems in Wireless Ad Hoc Sensor Networks, accepted to appear in Computer Communications, special issue on Sensor Networks.

5. J. Carle and D. Simplot, Energy Efficient Area Monitoring by Sensor Networks, IEEE Computer, Vol 37, No 2, pg. 40-46, 2004.

6. M. R. Garey and D. S. Johnson, Computers and Intractability: A guide to the theory of NPcompleteness, W. H. Freeman, 1979.

7. C.-F. Huang and Y.-C. Tseng, The Coverage Problem in a Wireless Sensor Network, ACM MobiCom'03, pg. 115-121, Sep. 2003.

8. D. Tian and N. D. Georganas, A Coverage-Preserving Node Scheduling Scheme for Large Wireless Sensor Networks, Proc. of the 1st ACM Workshop on Wireless Sensor Networks and Applications, pg. 32-41, 2002.

9. X. Wang, G. Xing, Y. Zhang, C. Lu, R. Pless, and C. D. Gill, Integrated Coverage and Connectivity Configuration in Wireless Sensor Networks, First ACM Conference on Embedded Networked Sensor Systems, pg. 28-39, 2003.

10. J. Wu and S. Yang, Coverage and Connectivity in Sensor Networks with Adjustable Ranges, International Workshop on Mobile and Wireless Networking (MWN), Aug. 2004.

11. Y. Ye, An $o\left(n^{3} l\right)$ Potential Reduction Algorithm for Linear Programming, Mathematical Programming, Vol 50, pg. 239-258, 1991.

12. H. Zhang, J. C. Hou, Maintaining Coverage and Connectivity in Large Sensor Networks, The Wireless Ad Hoc and Sensor Networks: An International Journal, 2005

13. F. Dai and J. Wu, Distributed Dominant Pruning in Ad Hoc Networks, in Proceedings of the IEEE 2003 International Conference on Communications (ICC 2003), Vol. 1, pg. 353-357, May 2003 Anchorage, AK.

14. X. Wang, G. Xing, Y. Zhang, C. Lu, R. Pless, and C. Gill, Integrated coverage and connectivity configuration in wireless sensor networks. In SenSys '03: Proceedings of the 1st International Conference on Embedded Networked Sensor Systems, pg. 28-39, Los Angeles, California, USA.

15. S. Shakkottai, R. Srikant, and N. Shroff. Unreliable sensor grids: coverage, connectivity and diameter. In IEEE INFOCOM, pg.1073-1083, 2003.

16. http://www.migatron.com/products/rps-400-6/rps-400-6.htm 